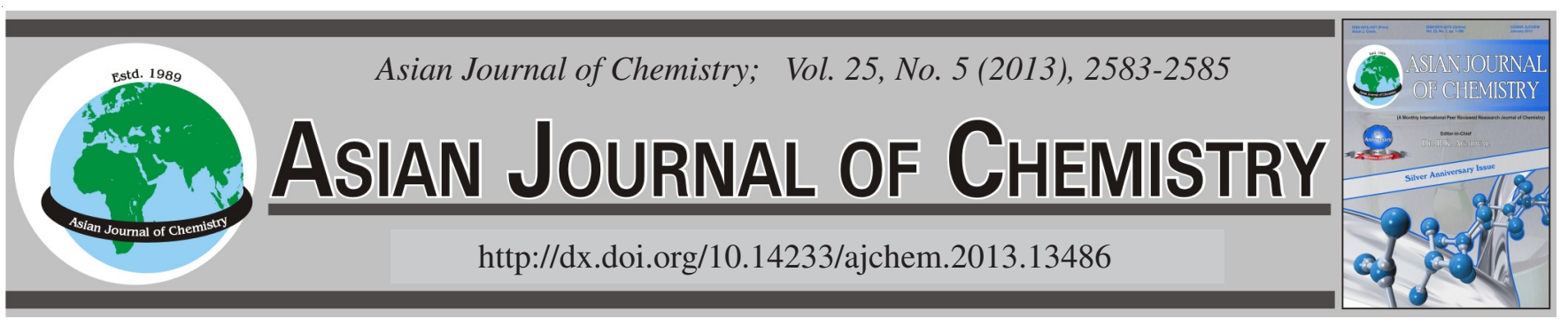

\title{
Synthesis and Characterization of Uniform ZnS Sphere Crystalline by Using a Zinc Coordination Complex as Precursor
}

\author{
Weinua Chen ${ }^{1}$, Qi Ding ${ }^{1,2}$, Shanshan Guo ${ }^{2}$, Yunanfang Chen ${ }^{1,2}, Z_{\text {hen LI }}^{1,2}$, Liwei Mi ${ }^{2, *}$ and Zhi ZhenG ${ }^{2, *}$
}

${ }^{1}$ College of Chemistry and Molecular Engineering, Zhengzhou University, Zhengzhou 450001, Henan Province, P.R. China

${ }^{2}$ Institute of Surface Micro and Nano Materials, Xuchang University, Xuchang 461000, Henan Province, P.R. China

*Corresponding authors: Fax: +86 374 2968784; Tel: +86 374 2968784; E-mail: mlwzzu@ 163.com; zhengzhi9999@yahoo.com.cn

Without using any surfactant or chemical assistant, we just use $\left[\mathrm{Zn}(\text { phen })_{2}\left(\mathrm{H}_{2} \mathrm{O}\right)_{2}\right]_{2} \mathrm{~L} \cdot 6 \mathrm{H}_{2} \mathrm{O}$ complex as precursor to react with sulfourea as sulphur source under moderate conditions. Uniform ZnS sphere crystalline has been successfully synthesized via a convenient solvothermal method, in which ethanediol and ethanediamine were utilized as mixed solvent. The composition and morphology of as-prepared samples were, respectively characterized by X-ray diffraction and scanning electron microscope. Also the photoluminescence property of the assynthesized ZnS materials was studied in this work.

Key Words: ZnS, Crystalline sphere, Photoluminescence.

\section{INTRODUCTION}

Along with technology advanced, semiconductor materials with micro or nano structure have induced more and more attention. Zinc sulphide is one of the very important semiconductors as a direct wide band gap compound and with a wide range of applications of optical materials ${ }^{1-3}$, quantum dot light-emitting devices ${ }^{4}$, LEDs materials ${ }^{5}$, etc. Until now, zinc sulphide was usually prepared by high temperature gas chromatography method and wet chemical method. It is well known that the wet chemical method contains hydrothermal process and solvothermal method ${ }^{6,7}$. Solvothermal method often be used to controlled synthesis of uniform micro or nano materials with special structures. For example, Fang's group has obtained zinc sulphide nanoplates with wide length less than $10 \mu \mathrm{m}$ on a silicon wafer which plated gold film with 40 $\mathrm{nm}$ thickness via firing made-up zinc sulphide ${ }^{8}$ and $\mathrm{Wu}$ and his co-workers have successfully synthesized zinc sulphide nanospheres through the use of hydrothermal method ${ }^{9}$.

In this study, our strategy for creating uniform $\mathrm{ZnS}$ sphere crystalline utilizes zinc coordination compound as precursor because such complex have already been proved to be promising materials for the synthesis of transition metal sulphides with special structure and shape $\mathrm{e}^{10,11}$, meanwhile, this kind complex also can easily be prepared under moderate conditions. Thus, a simple coordination complex $\left[\mathrm{Zn}(\text { phen })_{2}\left(\mathrm{H}_{2} \mathrm{O}\right)_{2}\right]_{2} \mathrm{~L}$. $6 \mathrm{H}_{2} \mathrm{O}$ was prepared, which was used as precursor for the purpose to control the concentration and release rate of $\mathrm{Zn}^{2+}$ ions. This study is believed to benefit for crystals growth. Uniform $\mathrm{ZnS}$ sphere crystalline was prepared under a convenient solvothermal method, in which ethanediol and ethanediamine were utilized as mixed solvent. Also the photoluminescence property of the as-synthesized $\mathrm{ZnS}$ materials was studied in this work.

\section{EXPERIMENTAL}

Sample preparation: All chemical reagents have been utilized in the present study were of analytical grade and used without further purification.

$\left[\mathrm{Zn}(\text { phen })_{2}\left(\mathrm{H}_{2} \mathrm{O}\right)_{2}\right] 2 \mathrm{~L} \cdot 6 \mathrm{H}_{2} \mathrm{O}+\mathrm{CS}\left(\mathrm{NH}_{2}\right)_{2} \underset{\text { ethanediol, ethanediamine }}{\triangle} \mathrm{ZnS}$

Coordination compound $\left[\mathrm{Zn}(\mathrm{phen})_{2}\left(\mathrm{H}_{2} \mathrm{O}\right)_{2}\right]_{2} \mathrm{~L} \cdot 6 \mathrm{H}_{2} \mathrm{O}$ was employed as precursor according to the previous report ${ }^{12}$. Asprepared $\left.\mathrm{Zn}(\text { phen })_{2}\left(\mathrm{H}_{2} \mathrm{O}\right)_{2}\right]_{2} \mathrm{~L} \cdot 6 \mathrm{H}_{2} \mathrm{O}(0.5 \mathrm{mmol})$ and sulfourea $(0.5 \mathrm{mmol})$ were added into the mixed solution of $8 \mathrm{~mL}$ ethanediol and $8 \mathrm{~mL}$ ethanediamine, then stirred for more than one hour. After this pretreatment process, the mixed solution was transferred into a Teflon-lined autoclave with $20 \mathrm{~mL}$ capacity. Then we sealed the autoclave and heated it to $160^{\circ} \mathrm{C}$, kept it at $160{ }^{\circ} \mathrm{C}$ for $24 \mathrm{~h}$. The autoclave was cooled to room temperature naturally. The resulting white products were collected and washed with distilled water and ethanol for several times and finally dried in vacuum at $50^{\circ} \mathrm{C}$ for $8 \mathrm{~h}$. 
Characterization: X-Ray diffraction (XRD) patterns were obtained on a Bruker D8 Advance X-ray powder diffractometer via using $\mathrm{CuK}_{\alpha}$ irradiation at a scan rate of $0.1 \%$ $\mathrm{s}$, measurements were carried out in the range of $20^{\circ} \leq 2 \theta \leq$ $80^{\circ}$. The morphology of the product prepared was examined by Zeiss Evo LS-15 scanning electron microscopy (SEM).

\section{RESULTS AND DISCUSSION}

The structure of the as-prepared $\mathrm{ZnS}$ sphere crystalline was determined by X-ray diffraction. As shown in Fig. 1, the peaks in the XRD spectrum of the as-grown sample can be ascribed to single crystalline zinc sulphide with wurtzite structure, JCPDS No. 36-1450. No peaks of other impurities were detected in the experimental error range, indicating the high purity of the obtained sample. The two theta degree of 26.91, $28.5,30.53,39.61,47.56,51.78,56.39$ correspond to the direction of 100, 002,101,102,110, 103 and 112, respectively.

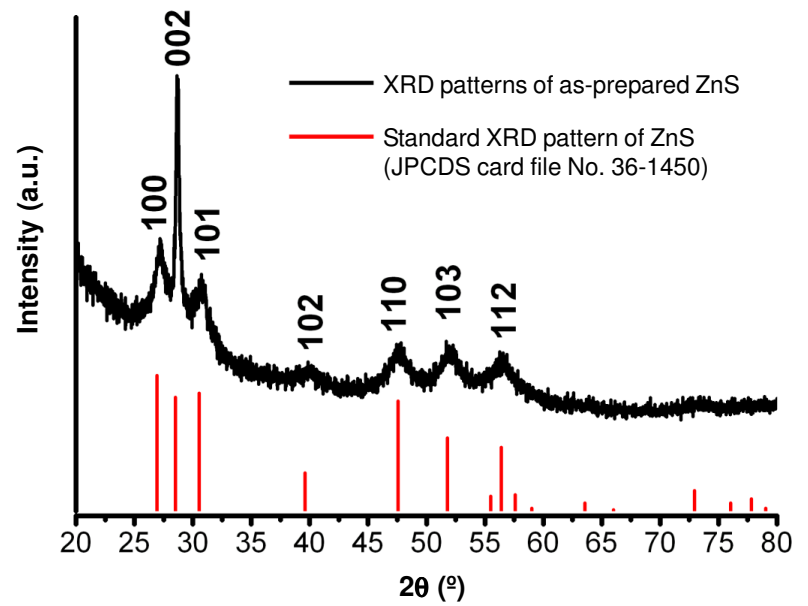

Fig. 1. XRD pattern of as-prepared $\mathrm{ZnS}$ sphere crystalline, the stick pattern is the standard pattern for $\mathrm{ZnS}$ with hexagonal wurtzite structure (JCPDS card file No. 36-1450)

The morphology and size of the as-synthesized products were characterized by scanning electron microscopy. As shown in Fig. 2a-d, the low magnification SEM images of the as-prepared $\mathrm{ZnS}$ microcrystals obtained at $160{ }^{\circ} \mathrm{C}$ for $24 \mathrm{~h}$, indicated that from which a large amount of ball-like $\mathrm{ZnS}$ microcrystals with diameters in the range of 0.4-0.5 $\mu \mathrm{m}$.

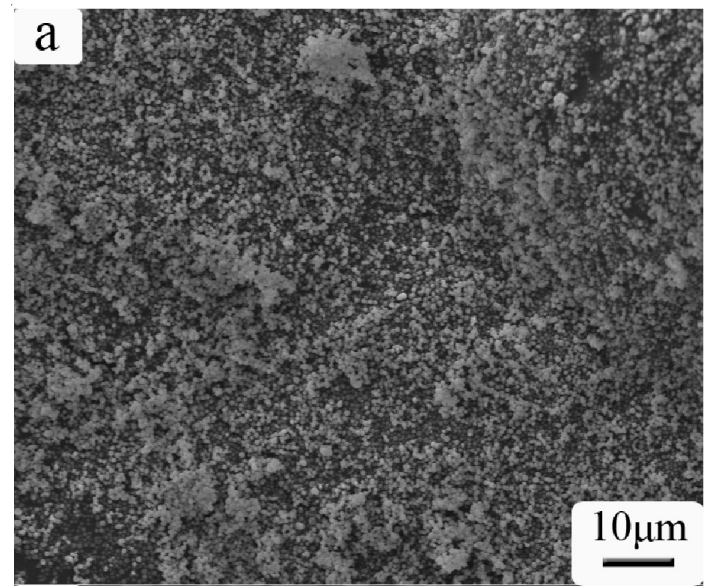

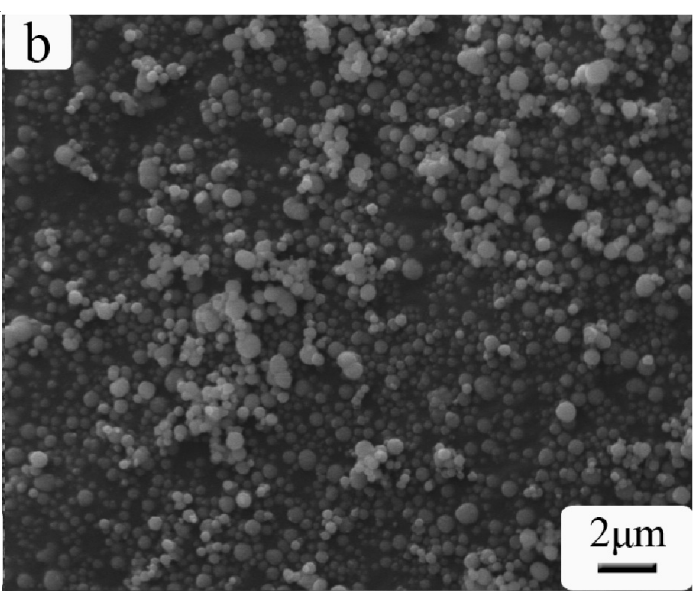
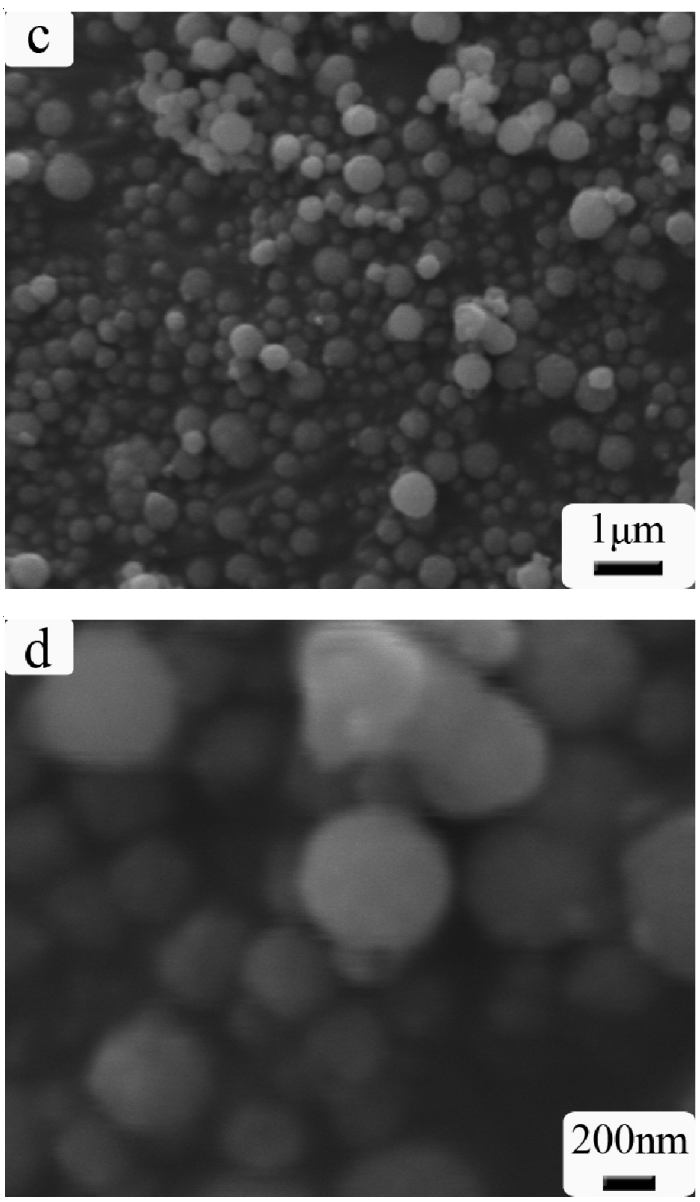

Fig. 2. Different magnification SEM images of as-prepared ZnS: a for 1000 times; b for 3000 times; c for 10000 times and d for 40000 times

The photoluminescence property of as-prepared ball-like $\mathrm{ZnS}$ was also studied. Fig. 3 gives the room temperature fluorescence spectrum of the sample under an excitation $\lambda_{\mathrm{ex}}=$ $250 \mathrm{~nm}$. There is an emission band with a two-peak structure. This band could be Gaussian divided into two luminescent peaks at $c a .294$ and $307 \mathrm{~nm}$.

\section{Conclusion}

We have succeeded in synthesizing uniform $\mathrm{ZnS}$ microcrystals just by applying $\left[\mathrm{Zn}(\text { phen })_{2}\left(\mathrm{H}_{2} \mathrm{O}\right)_{2}\right]_{2} \mathrm{~L} \cdot 6 \mathrm{H}_{2} \mathrm{O}$ and sulfourea as the precursor of $\mathrm{Zn}^{2+}$ and sulphide source via a solvothermal method. This preparation method is an environmentally friendly and low cost. 


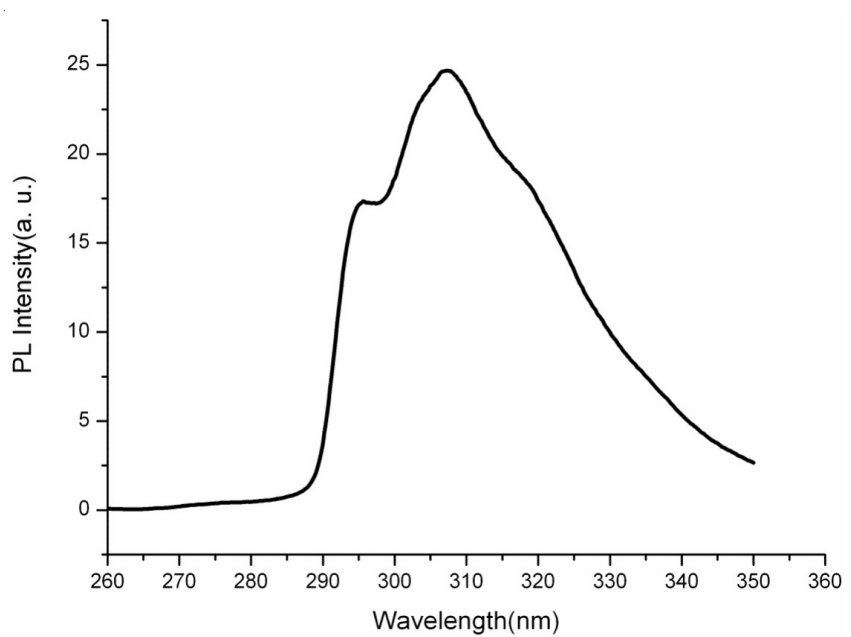

Fig. 3. Photoluminescence property of as-prepared $\mathrm{ZnS}$

\section{ACKNOWLEDGEMENTS}

This work was supported by the National Science Foundation of China (Grant No. 21001090 and 21103153), China Postdoctoral Science Foundation funded project (Grant No. 20100480856 and 201003398), NCET-08-0665 and Henan Province (Grant No. 2010HASTIT018 and 2012TRTSTHN021).

\section{REFERENCES}

1. R. Chen, D.H. Li, B. Liu, Z.P. Peng, G.G. Gurzadyan, Q.H. Xiong and H.D. Sun, Nano Lett., 10, 4956 (2010).

2. J.S. Schrie, D.O. Demchenko and L.W. Wang, Nano Lett., 7, 2377 (2007).

3. C. Meng, Y. Xiao, P. Wang, L. Zhang, Y.X. Liu and L.M. Tong, Adv. Mater., 23, 3770 (2011).

4. E.M. Likovich, R. Jaramillo K.J. Russell, S. Ramanathan and V. Narayanamurti, Adv. Mater., 23, 4521 (2011).

5. K. Kim, J.Y. Woo, S.H. Jeong and C.S. Han, Adv. Mater., 23, 911 (2011).

6. G.Z. Shen, Y. Bando and D. Golberg, Appl. Phys. Lett., 88, 107 (2006).

7. Y. Jiang, X.M. Meng, J. Liu, Z.R. Hong, C.S. Lee and S.T. Lee, Adv. Mater., 15, 1195 (2003).

8. X.S. Fang, C.H. Ye, L.D. Zhang, Y.H. Wang and Y.C. Wu, Adv. Funct. Mater., 15, 63 (2005).

9. Q.Z. Wu, H.Q. Cao, S.C. Zhang, X.R. Zhang and D. Rabinovich, Inorg. Chem., 45, 7316 (2006).

10. L.W. Mi, M.L. Han, Z. Li, F.L. Yang, C.Y. Shen and Z. Zheng, Z. Anorg. Allg. Chem., 636, 1913 (2010).

11. L.W. Mi, M.L. Han, D.P. Li, W.H. Chen and Z. Zheng, Z. Anorg. Allg. Chem., 634, 273 (2008).

12. J. Yang, J.F. Ma, D.M. Wu, L.P. Guo and J.F. Liu, Transition Met. Chem., 28, 789 (2003). 